Notes on the commutativity of prime near-rings

\author{
Emine Koç
}




\title{
NOTES ON THE COMMUTATIVITY OF PRIME NEAR-RINGS
}

\author{
EMINE KOÇ
}

Received December 18, 2010

\begin{abstract}
Let $N$ be a 3-prime right near-ring and let $f$ be a generalized $(\theta, \theta)$ - derivation on $N$ with associated $(\theta, \theta)$-derivation $d$. It is proved that $N$ must be a commutative ring if $d \neq 0$ and one of the following conditions is satisfied for all $x, y \in N:(i) f([x, y])=0$; (ii) $f([x, y])=\theta([x, y])$; (iii) $f(x o y)=0 ;($ iv $) f(x o y)=\theta(x o y) ;(v) f([x, y])=\theta(x o y)$; (vi) $f(x$ oy $)=\theta([x, y])$. We also prove theorems which assert that $N$ is commutative, but not necessarily a ring.
\end{abstract}

2000 Mathematics Subject Classification: 16Y30

Keywords: near-rings, $(\theta, \theta)$-derivation, generalized $(\theta, \theta)$-derivation

\section{INTRODUCTION}

An additively written group $(N,+)$ equipped with a binary operation $\cdot: N \times N \rightarrow$ $N,(x, y) \rightarrow x y$ such that $(x y) z=x(y z)$ and $(x+y) z=x z+y z$ for all $x, y, z \in N$ is called a right near-ring. Recall that a near-ring $N$ is called 3 -prime if for any $x, y \in N, x N y=0$ implies that $x=0$ or $y=0$. For $x, y \in N$ the symbol $[x, y]$ will denote $x y-y x$, while the symbol $x o y$ will denote $x y+y x . Z$ is the multiplicative center of $N$. An additive mapping $d: N \rightarrow N$ is said to be a derivation if $d(x y)=x d(y)+d(x) y$ for all $x, y \in N$, or equivalently, as noted in [12], that $d(x y)=d(x) y+x d(y)$ for all $x, y \in N$. Recently, in [7], Bresar defined the following concept. An additive mapping $F: N \rightarrow N$ is called a generalized derivation if there exists a derivation $d: N \rightarrow N$ such that

$$
F(x y)=F(x) y+x d(y) \text {, for all } x, y \in N .
$$

Basic examples are derivations and generalized inner derivations (i.e., maps of type $x \rightarrow a x+x b$ for some $a, b \in N)$. One may observe that the concept of generalized derivations includes the concept of derivations and of left multipliers (i.e., $F(x y)=$ $F(x) y$, for all $x, y \in N)$.

Inspired by the definition of derivation (resp. generalized derivation), we define the notion of $(\theta, \phi)$-derivation (resp. generalized $(\theta, \phi)$-derivation) as follows: Let $\theta, \phi$ be two near-ring automorphisms of $N$. An additive mapping $d: N \rightarrow N$ is called a $(\theta, \phi)$-derivation (resp. generalized $(\theta, \phi)$-derivation) if $d(x y)=\phi(x) d(y)+$ 
$d(x) \theta(y)$ (resp. $f(x y)=f(x) \theta(y)+\phi(x) d(y)$, where $d$ is a $(\theta, \phi)$-derivation) holds for all $x, y \in N$. It is noted that $d(x y)=d(x) \theta(y)+\phi(x) d(y)$, for all $x, y \in$ $N$ in $[9$, Lemma 1]. Of course a $(1,1)$-derivation (resp. generalized $(1,1)-$ derivation ) is a derivation (resp. generalized derivation) on $N$, where 1 is the identity on $N$.

Many authors have investigated the properties of derivations of prime and semiprime rings. The study of derivations of near-rings was initiated by H. E. Bell and G. Mason in 1987 [5]. Some recent results on rings deal with commutativity on prime and semiprime rings admitting suitably constrained derivations. It is natural to look for comparable results on near-rings and this has been done in [3], [5], [6], [4], [2], [9].

In [8], Daif and Bell showed that the ideal $I$ of a semiprime ring is contained in the center of $R$ if

$$
d([x, y])=[x, y] \text { for all } x, y \in I \text { or } d([x, y])=-[x, y] \text { for all } x, y \in I .
$$

Several authors have obtained commutativity results for prime or semiprime rings admitting derivations or generalized derivations $d$ satisfying (1.1) or similar conditions (see [1], [11], [10]). The first purpose of this paper is to show that 3-prime near-rings must be commutative rings if they admit appropriate generalized $(\theta, \theta)-$ derivations satisfying conditions related to (1.1). The second aim is to prove some commutativity theorems for 3 -prime near-rings with $(\theta, \theta)$-derivations.

\section{RESULTS ON GENERALIZED $(\theta, \theta)$-DERIVATIONS}

Lemma 1. [9, Theorem 2] Let $N$ be a 3-prime near-ring admitting a non trivial $(\sigma, \tau)$-derivation $d$. If $d(N) \subset Z$, then $(N,+)$ is abelian. Moreover, if $N$ is 2torsion free and $\sigma, \tau$ commute with $d$, then $N$ is a commutative ring.

Theorem 1. Let $N$ be a 2-torsion free 3-prime near-ring, $(f, d)$ a generalized $(\theta, \theta)-$ derivation of $N$ and $d \theta=\theta d$. If $f([x, y])=0$ for all $x, y \in N$ and $d \neq 0$, then $N$ is a commutative ring.

Proof. By the hyphothesis, we have

$$
f([x, y])=0, \text { for all } x, y \in N .
$$

Replacing $y$ by $y x$ in (2.1) and using $[x, y x]=[x, y] x$, we obtain that

$$
f([x, y]) \theta(x)+\theta([x, y]) d(x)=0, \text { for all } x, y \in N .
$$

By (2.1), we get

$$
\theta([x, y]) d(x)=0, \text { for all } x, y \in N,
$$

and so

$$
\theta(x) \theta(y) d(x)=\theta(y) \theta(x) d(x), \text { for all } x, y \in N .
$$

Taking $z y, z \in N$ instead of $y$ in (2.2) and using (2.2), we arrive at

$$
\theta([x, z]) \theta(y) d(x)=0 \text {, for all } x, y, z \in N \text {. }
$$


Since $\theta$ is an automorphism of $N$, we have

$$
\theta([x, z]) N d(x)=0, \text { for all } x, z \in N .
$$

By the primeness of $N$, we get either $\theta([x, z])=0$ or $d(x)=0$ for each $x \in N$. Again using $\theta \in A u t N$, we conclude that

$$
x \in Z \text { or } d(x)=0 \text { for each } x \in N .
$$

If $x \in Z$, then $d(x) \in Z$. Indeed, for all $y \in N$, we get

$$
x y=y x,
$$

and so

$$
\begin{aligned}
d(x y) & =d(y x), \text { for all } y \in N, \\
\theta(x) d(y)+d(x) \theta(y) & =d(y) \theta(x)+\theta(y) d(x), \text { for all } y \in N .
\end{aligned}
$$

Using $x \in Z$ in this equation, we obtain that

$$
d(x) \theta(y)=\theta(y) d(x), \text { for all } y \in N
$$

and so

$$
d(x) y=y d(x), \text { for all } y \in N .
$$

Thus $d(x) \in Z$, for all $x \in N$.

By Lemma 1 , we conclude that $N$ is a commutative ring. This completes the proof.

Theorem 2. Let $N$ be a 2-torsion free 3-prime near-ring, $(f, d)$ a generalized $(\theta, \theta)-$ derivation of $N$ and $d \theta=\theta d$. If $f([x, y])= \pm \theta([x, y])$ for all $x, y \in N$ and $d \neq 0$, then $N$ is a commutative ring.

Proof. Replacing $y$ by $y x$ in the hypothesis yields that

$$
f([x, y] x)= \pm \theta([x, y] x) \text {, for all } x, y \in N,
$$

and so

$$
f([x, y]) \theta(x)+\theta([x, y]) d(x)= \pm \theta([x, y]) \theta(x), \text { for all } x, y \in N .
$$

Using our hypothesis, the above relation yields that

$$
\pm \theta([x, y]) \theta(x)+\theta([x, y]) d(x)= \pm \theta([x, y]) \theta(x), \text { for all } x, y \in N,
$$

and so

$$
\theta([x, y]) d(x)=0, \text { for all } x, y \in N .
$$

Arguing in the similar manner as we have done in the proof of Theorem 1, we find $N$ is a commutative ring.

Theorem 3. Let $N$ be a 2-torsion free 3-prime near-ring, $(f, d)$ a generalized $(\theta, \theta)-$ derivation of $N$ and $d \theta=\theta d$. If $f(x \circ y)=0$ for all $x, y \in N$ and $d \neq 0$, then $N$ is a commutative ring. 
Proof. Assume that

$$
f(x \circ y)=0, \text { for all } x, y \in N .
$$

Substituting $y x$ for $y$ in (2.3), we get

$$
f(x \circ y) \theta(x)+\theta(x o y) d(x)=0 \text {, for all } x, y \in N .
$$

By (2.3), we obtain that

$$
\theta(x o y) d(x)=0, \text { for all } x, y \in N,
$$

and so

$$
\theta(x) \theta(y) d(x)=-\theta(y) \theta(x) d(x), \text { for all } x, y \in N .
$$

Taking $z y, z \in N$ instead of $y$ in this relation and using this equation, we have

$$
\theta(x) \theta(z) \theta(y) d(x)=\theta(z) \theta(x) \theta(y) d(x), \text { for all } x, y, z \in N,
$$

and so

$$
\theta([x, z]) \theta(y) d(x)=0, \text { for all } x, y, z \in N .
$$

Applying the same techniques in the proof of Theorem 1, we conclude that $N$ is a commutative ring.

Theorem 4. Let $N$ be a 2-torsion free 3-prime near-ring, $(f, d)$ a generalized $(\theta, \theta)-$ derivation of $N$ and $d \theta=\theta d$. If $f(x o y)= \pm \theta$ (xoy) for all $x, y \in N$ and $d \neq 0$, then $N$ is commutative ring.

Proof. We have

$$
f(x o y)= \pm \theta(x o y), \text { for all } x, y \in N .
$$

Substituting $y x$ for $y$ in (2.4), we obtain that

$$
f(x \circ y) \theta(x)+\theta(x o y) d(x)= \pm \theta(x o y) \theta(x), \text { for all } x, y \in N .
$$

Using (2.4), we get

$$
\theta(x o y) d(x)=0, \text { for all } x, y \in N .
$$

Replacing $y$ by $z y$ in the above relation, we arrive at

$$
\theta([x, z]) \theta(y) d(x)=0 \text {, for all } x, y, z \in N .
$$

Again using the same arguments in the proof of Theorem 1, we find the required result.

Theorem 5. Let $N$ be a 2-torsion free 3-prime near-ring, $(f, d)$ a generalized $(\theta, \theta)-$ derivation of $N$ and $d \theta=\theta d$. If $f([x, y])= \pm \theta(x o y)$, for all $x, y \in N$ and $d \neq 0$, then $N$ is a commutative ring. 
Proof. Writing $y x$ by $y$ in the hypothesis, we have

$$
f([x, y]) \theta(x)+\theta([x, y]) d(x)= \pm \theta(x o y) \theta(x), \text { for all } x, y \in N,
$$

and so

$$
\pm \theta(x \circ y) \theta(x)+\theta([x, y]) d(x)= \pm \theta(x \circ y) \theta(x), \text { for all } x, y \in N .
$$

That is

$$
\theta([x, y]) d(x)=0, \text { for all } x, y \in N .
$$

Using the same arguments in the proof of Theorem 1, we arrive at the required result.

Theorem 6. Let $N$ be a 2-torsion free 3-prime near-ring, $(f, d)$ a generalized $(\theta, \theta)-$ derivation of $N$ and $d \theta=\theta$ d. If $f(x \circ y)= \pm \theta([x, y])$ for all $x, y \in N$ and $d \neq 0$, then $N$ is a commutative ring.

Proof. Suppose that

$$
f(x \circ y)= \pm \theta([x, y]), \text { for all } x, y \in N .
$$

Replacing $y$ by $y x$ in this equation gives that

$$
f(x \circ y) \theta(x)+\theta(x o y) d(x)= \pm \theta([x, y]) \theta(x), \text { for all } x, y \in N .
$$

By the hypothesis, we have

$$
\pm \theta([x, y]) \theta(x)+\theta(x o y) d(x)= \pm \theta([x, y]) \theta(x), \text { for all } x, y \in N,
$$

and so

$$
\theta(x o y) d(x)=0, \text { for all } x, y \in N .
$$

Arguing in the similar manner as we have done in the proof of Theorem 3, we conclude that $N$ is a commutative ring.

Remark 1. Each of the above theorems yields on obvious result for $(\theta, \theta)$-derivations.

\section{RESUlts ON $(\theta, \theta)$-DERIVATIONS}

Lemma 2. Let $N$ be a right near-ring, $d a(\theta, \theta)$-derivation of $N$ and $a \in N$. Then

$$
a(d(x) \theta(y)+\theta(x) d(y))=a d(x) \theta(y)+a \theta(x) d(y), \text { for all } x, y \in N .
$$

Proof. Given $x, y \in N$, obtain

$$
\begin{aligned}
d(a(x y)) & =d(a) \theta(x y)+\theta(a) d(x y) \\
& =d(a) \theta(x) \theta(y)+\theta(a)(d(x) \theta(y)+\theta(x) d(y)) .
\end{aligned}
$$

On the other hand,

$$
\begin{aligned}
d((a x) y) & =d(a x) \theta(y)+\theta(a x) d(y) \\
& =d(a) \theta(x) \theta(y)+\theta(a) d(x) \theta(y)+\theta(a) \theta(x) d(y) .
\end{aligned}
$$


Comparing (3.1) and (3.2), we conclude that

$$
\theta(a)(d(x) \theta(y)+\theta(x) d(y))=\theta(a) d(x) \theta(y)+\theta(a) \theta(x) d(y),
$$

for all $x, y \in N$. Since $\theta$ is an automorphism of $N$, we can write this equation as

$$
a(d(x) \theta(y)+\theta(x) d(y))=a d(x) \theta(y)+a \theta(x) d(y), \text { for all } x, y \in N .
$$

Theorem 7. Let $N$ be a 2-torsion free 3-prime near-ring, $d$ a $(\theta, \theta)$-derivation of $N$. If $d(x) d(y)=\theta([x, y])$ for all $x, y \in N$, then $N$ is commutative.

Proof. Assume that

$$
d(x) d(y)=\theta([x, y]), \text { for all } x, y \in N .
$$

Replacing $y$ by $y x$ in (3.3), we obtain that

$$
d(x)(d(y) \theta(x)+\theta(y) d(x))=\theta([x, y]) \theta(x) \text {, for all } x, y \in N .
$$

By Lemma 2, we have

$$
d(x) d(y) \theta(x)+d(x) \theta(y) d(x)=\theta([x, y]) \theta(x), \text { for all } x, y \in N .
$$

Using equation (3.3), we find that

$$
\theta([x, y]) \theta(x)+d(x) \theta(y) d(x)=\theta([x, y]) \theta(x), \text { for all } x, y \in N,
$$

and so

$$
d(x) \theta(y) d(x)=0, \text { for all } x, y \in N .
$$

Since $\theta$ is an automorphism of $N$, we get

$$
d(x) N d(x)=0 \text {, for all } x \in N .
$$

By the primeness of $N$, we arrive at $d(x)=0$, for all $x \in N$. If $d=0$, then we have $\theta([x, y])=0$ for all $x, y \in N$ by the hypothesis, and so $N$ is commutative.

Theorem 8. Let $N$ be a 2-torsion free 3-prime near-ring, $d$ a $(\theta, \theta)$-derivation of $N$. If $d(x) d(y)=\theta$ (xoy) for all $x, y \in N$, then $N$ is commutative.

Proof. Replacing $y$ by $y x$ in the hypothesis, we have

$$
d(x)(d(y) \theta(x)+\theta(y) d(x))=\theta(x \circ y) \theta(x), \text { for all } x, y \in N .
$$

By Lemma 2, we get

$$
d(x) d(y) \theta(x)+d(x) \theta(y) d(x)=\theta(x o y) \theta(x), \text { for all } x, y \in N .
$$

Using the hypothesis, we obtain that

$$
\theta(x \circ y) \theta(x)+d(x) \theta(y) d(x)=\theta(x o y) \theta(x), \text { for all } x, y \in N .
$$

and so

$$
d(x) \theta(y) d(x)=0, \text { for all } x, y \in N .
$$


Since $\theta$ is an automorphism of $N$, we get

$$
d(x) N d(x)=0 \text {, for all } x \in N .
$$

By the primeness of $N$, we obtain that $d=0$. If $d=0$, then we have $\theta(x o y)=0$, for all $x, y \in N$ by the hypothesis, and so $x y=-y x$, for all $x, y \in N$. Writing $y z$ by $y$ in this equation, we have

$$
x y z=-y z x=y x z, \text { for all } x, y, z \in N,
$$

and so

$$
[x, y] z=0, \text { for all } x, y, z \in N .
$$

Since $N$ is a $3-$ prime near-ring, we get $[x, y]=0$, for all $x, y \in N$, and so, $N$ is commutative.

Theorem 9. Let $N$ be a 2-torsion free 3-prime near-ring and $d$, $h$ be two $(\theta, \theta)-$ derivations. If $d(x) \theta(y)=\theta(x) h(y)$ for all $x, y \in N$, then $d=h=0$.

Proof. We get

$$
d(x) \theta(y)=\theta(x) h(y), \text { for all } x, y \in N .
$$

Replacing $y$ by $y z, z \in N$ in (3.4), we arrive at

$$
d(x) \theta(y) \theta(z)=\theta(x)(h(y) \theta(z)+\theta(y) h(z)), \text { for all } x, y, z \in N .
$$

By Lemma 2, we have

$$
d(x) \theta(y) \theta(z)=\theta(x) h(y) \theta(z)+\theta(x) \theta(y) h(z), \text { for all } x, y, z \in N .
$$

Using (3.4), we find that

$$
\theta(x) h(y) \theta(z)=\theta(x) h(y) \theta(z)+\theta(x) \theta(y) h(z), \text { for all } x, y, z \in N,
$$

and so

$$
\theta(x) \theta(y) h(z)=0, \text { for all } x, y, z \in N \text {. }
$$

That is

$$
\theta(x) N h(z)=0 \text {, for all } x, z \in N \text {. }
$$

By the primeness of $N$ gives $h=0$. If $h=0$, then $d(x) \theta(y)=0$, for all $x, y \in N$ by the hypothesis. Again using the primeness of $N$, we get $d=0$. This completes the proof. 


\section{REFERENCES}

[1] N. Argaç, "On prime and semiprime rings with derivations," Algebra Colloq., vol. 13, no. 3, pp. 371-380, 2006.

[2] M. Ashraf, A. Ali, and S. Ali, “( $\sigma, \tau)$-derivations on prime near rings," Arch. Math. (Brno), vol. 40, no. 3, pp. 281-286, 2004.

[3] K. I. Beidar, Y. Fong, and X. K. Wang, "Posner and Herstein theorems for derivations of 3-prime near-rings," Comm. Algebra, vol. 24, no. 5, pp. 1581-1589, 1996.

[4] H. E. Bell and N. Argaç, "Derivations, products of derivations, and commutativity in near-rings," Algebra Colloq., vol. 8, no. 4, pp. 399-407, 2001.

[5] H. E. Bell and G. Mason, "On derivations in near-rings," in Near-rings and near-fields, ser. NorthHolland Math. Stud., vol. 137. Amsterdam: North-Holland, 1987, pp. 31-35.

[6] H. E. Bell and G. Mason, "On derivations in near-rings and rings," Math. J. Okayama Univ., vol. 34, pp. 135-144, 1992.

[7] M. Brešar, "On the distance of the composition of two derivations to the generalized derivations," Glasgow Math. J., vol. 33, no. 1, pp. 89-93, 1991.

[8] M. N. Daif and H. E. Bell, "Remarks on derivations on semiprime rings," Internat. J. Math. Math. Sci., vol. 15, no. 1, pp. 205-206, 1992.

[9] O. Gölbaşi, "Some properties of prime near-rings with $(\sigma, \tau)$-derivation," Siberian Math. J., vol. 46, no. 2, pp. 270-273, 2005.

[10] O. Gölbaşi, "On commutativity of semiprime rings with generalized derivations," Indian J. Pure Appl. Math., vol. 40, no. 3, pp. 191-199, 2009.

[11] M. A. Quadri, M. S. Khan, and N. Rehman, "Generalized derivations and commutativity of prime rings," Indian J. Pure Appl. Math., vol. 34, no. 9, pp. 1393-1396, 2003.

[12] X. K. Wang, "Derivations in prime near-rings," Proc. Amer. Math. Soc., vol. 121, no. 2, pp. 361366, 1994.

\section{Author's address}

\section{Emine Koç}

Cumhuriyet University, Faculty of Science, Department of Mathematics, Sivas - TURKEY

E-mail address: eminekocecumhuriyet.edu.tr

URL: http://www.cumhuriyet.edu.tr 